\title{
On the Real Cubic Fields
}

\author{
By P. Llorente and A. V. Oneto
}

\begin{abstract}
In this paper the authors announce a table of the 4753 totally real nonconjugate nonabelian cubic fields with discriminant less than 100000 . Each field is given by its discriminant, the coefficients of a generating polynomial and the index of this polynomial over the field. A basis of the integers of the field is also given. Some differences with other tables are pointed out.
\end{abstract}

Godwin and Samet [3] have described the construction of a table of real cubic fields with discriminants $D<20000$. Using similar methods Angell [1] extends these results up to $D<100000$. Using a different method the authors [5] have constructed a table of the 4753 real nonabelian cubic fields with discriminants $D<100000$. Here and in the sequel triplets of conjugate fields are counted once only.

The method developed in [5] generalizes the one used in [4] and the table constructed there gives, for each cubic field $K$, its discriminant, an irreducible polynomial $f(X)$ which defines $K$, the index of $f(X)$ over $K$ and a basis for the integers of $K$.

In this work we present some consequences derived from [5]. In particular we have discovered that ten fields are missing from Angell's table. In Table 1 we give each of these ten fields and its corresponding class numbers $h$. The field $K$, with discriminant $D$, is generated by a root $\theta$ of the polynomial $f(X)=X^{3}-A X+B ; S$ is the index of $\theta$, and $\{1, \theta, \alpha\}$ is a basis of the integers of $K$, where

$$
\alpha=\frac{\theta^{2}+T \theta+\left(T^{2}-A\right)}{S} .
$$

TABLE 1

\begin{tabular}{|c|r|r|r|r|r|}
\hline$D$ & \multicolumn{1}{|c|}{$A$} & \multicolumn{1}{c|}{$B$} & $S$ & $T$ & $h$ \\
\hline 32404 & 64 & 194 & 1 & 0 & 1 \\
35996 & 167 & 552 & 17 & -4 & 1 \\
37108 & 76 & 244 & 2 & 0 & 2 \\
37133 & 167 & 374 & 20 & -7 & 1 \\
38905 & 73 & 147 & 5 & 1 & 1 \\
39992 & 65 & 198 & 1 & 0 & 1 \\
43165 & 163 & 482 & 16 & -7 & 1 \\
43173 & 30 & 49 & 1 & 0 & 1 \\
43176 & 138 & 576 & 6 & 0 & 1 \\
95484 & 183 & 936 & 3 & 0 & 1 \\
\hline
\end{tabular}

Received January 30, 1981; revised December 22, 1981.

1980 Mathematics Subject Classification. Primary 12A30, 12-04.

Key words and phrases. Real cubic field, class number. 
Table 1 allows the corrections of the results of [1], in particular the statistical table there given. However, as that table has some other mistakes, we prefer to reproduce it with its correct values in Table 2.

TABLE 2

\begin{tabular}{|r|c|c|c|c|c|c|c|c|c|c|}
\cline { 3 - 9 } \multicolumn{2}{c|}{} & \multicolumn{6}{|c|}{ Class Number } \\
\hline Bounds on $D$ & No. of fields & 1 & 2 & 3 & 4 & 5 & 6 & 7 & 8 & 9 \\
\hline 1 to 10000 & 382 & 358 & 9 & 14 & 1 & - & - & - & - & - \\
10001 to 20000 & 450 & 408 & 20 & 20 & 2 & - & - & - & - & - \\
20001 to 30000 & 467 & 415 & 26 & 21 & 2 & 2 & - & 1 & - & - \\
30001 to 40000 & 479 & 425 & 24 & 24 & 2 & 4 & - & - & - & - \\
40001 to 50000 & 485 & 418 & 29 & 33 & 3 & 1 & 1 & - & - & - \\
50001 to 60000 & 500 & 442 & 27 & 23 & 1 & 1 & 2 & 3 & 1 & - \\
60001 to 70000 & 490 & 417 & 32 & 33 & 3 & 4 & - & - & - & 1 \\
70001 to 80000 & 509 & 436 & 35 & 30 & 3 & 3 & 2 & - & - & - \\
80001 to 90000 & 514 & 432 & 44 & 33 & 2 & 2 & - & 1 & - & - \\
90001 to 100000 & 528 & 442 & 42 & 37 & 1 & 2 & 2 & 2 & - & - \\
\hline
\end{tabular}

Let $N(x)$ be the number of nonabelian real cubic fields with discriminant $D<x$, $P(x)$ the number of real cubic fields (abelian and nonabelian) with discriminant $D<x$, and

$$
c(x)=\frac{P(x)}{x} \pi^{2}
$$

In [2] it is proved that

$$
\lim _{x \rightarrow \infty} c(x) \geqslant 1 / 240 \text { and } \varlimsup_{x \rightarrow \infty} c(x) \leqslant 5 / 4
$$

and it is observed that both constants could be improved. In Table 3 we give the values of $N(x), P(x)$ and $c(x)$ for several values of $x$.

TABLE 3

\begin{tabular}{|c|r|r|r|}
\hline$x$ & $N(x)$ & $P(x)$ & $c(x)$ \\
\hline 10000 & 366 & 382 & 0.3770 \\
20000 & 808 & 832 & 0.4106 \\
30000 & 1270 & 1299 & 0.4274 \\
40000 & 1746 & 1778 & 0.4387 \\
50000 & 2227 & 2263 & 0.4467 \\
60000 & 2725 & 2763 & 0.4545 \\
70000 & 3211 & 3253 & 0.4587 \\
80000 & 3716 & 3762 & 0.4641 \\
90000 & 4229 & 4276 & 0.4689 \\
100000 & 4753 & 4804 & 0.4741 \\
\hline
\end{tabular}


We now give a sketch of the method employed (for details see [5]). Let $\bar{D}>0$ be an integer. We propose to determine all conjugate triplets of noncyclic cubic fields with discriminant $D$ such that $0<D \leqslant \bar{D}$.

Each of these triplets is defined by a polynomial $f(A, B, X)=X^{3}-A X+B$, where $A>0$ and $B>0$ are integers such that

(i) $f(A, B, X)$ is irreducible in $Q[X]$.

(ii) There is no integer $m>1$ such that $m^{2}\left|A, m^{3}\right| B$, and the discriminant of $f(A, B, X)$ is

$$
D(A, B)=4 A^{3}-27 B^{2}=D S^{2},
$$

where $S \geqslant 1$ is an integer.

We consider the congruences

$$
A \equiv 3(\bmod 9), \quad B \equiv \pm(A-1)(\bmod 27) .
$$

By Voronoi's theorem, if the congruences (1) are not satisfied, then there are integers $T, U$, and $V$ such that

(iii) $-S / 2<T \leqslant S / 2$,

(iv) $3 T^{2}-A=U S$,

(v) $T^{3}-A T+B=V S^{2}$.

(vi) If we replace $S$ by an integer $\bar{S}>S$ such that $\bar{S}^{2} \mid D(A, B)$, then there are no integers $T, U, V$ that satisfy the above-mentioned conditions.

If the congruences (1) are satisfied, then it follows that $S=27 S^{\prime}$, and there are integers $T, U, V$ such that

(vii) $-3 S^{\prime} / 2<T \leqslant 3 S^{\prime} / 2$,

(viii) $3 T^{2}-A=9 U S^{\prime}$,

(ix) $T^{3}-A T+B=27 V S^{\prime 2}$.

(x) If we replace $S^{\prime}$ by an integer $\overline{S^{\prime}}>S^{\prime}$ such that $\left(27 \overline{S^{\prime}}\right)^{2} \mid D(A, B)$, then there are no integers $T, U, V$ that satisfy the above conditions.

It follows that if we choose a minimal $T$, with each pair $(A, B)$ is associated a unique quadruple $(S, T, U, V)$ of integers that satisfies the above relations in each case. Also, each pair $(A, B)$ determines a binary quadratic form $F(A, B)$ defined in such a way that if $K(A, B)$ is a cubic field defined by $f(A, B, X)$, the minimal polynomial of a nonzero integer $\gamma$ of $K(A, B)$ with null trace is

$$
X^{3}-A^{\prime} X-N(\gamma)
$$

where $A^{\prime}$ is an integer represented by $F(A, B)$.

The coefficients of $F(A, B)$ are functions of $S, T, U, V$, and the discriminant of $F(A, B)$ is $-D / 3$ if the congruences (1) are not satisfied and $27 \mid D$, and it is $-3 D$ otherwise (see [5]).

Thus $F(A, B)$ is positive definite. Moreover, there is a pair $\left(A^{\prime}, B^{\prime}\right)$, which defines the same triplet of cubic fields as $(A, B)$ if and only if $A^{\prime}$ is a nonzero integer represented by $F(A, B)$, and in such a case the quadratic forms $F(A, B)$ and $F\left(A^{\prime}, B^{\prime}\right)$ represent the same integers.

These considerations allow us to assume that $A$ is the least integer represented by $F(A, B)$, and we obtain the bounds:

$$
4 \leqslant A \leqslant \sqrt{\bar{D}} \text { and } 1 \leqslant B<2 \frac{A}{3} \sqrt{\frac{A}{3}} .
$$


Using these bounds one can build up all pairs $(A, B)$ each of which determines a triplet of conjugate noncyclic cubic fields with discriminant $D$ such that $0<D \leqslant \bar{D}$.

It can occur that two different pairs $(A, B)$ and $\left(A^{\prime}, B^{\prime}\right)$ determine the same triplet of cubic fields. This happens, as is proved in [5], if and only if $F(A, B)$ and $F\left(A^{\prime}, B^{\prime}\right)$ are equivalent quadratic forms. This provides us with an easy way to eliminate pairs which determine the same triplet of cubic fields.

Postgrado de Ingenieria, PEAM

Universidad del Zulia

Apartado No. 98

Maracaibo, Venezuela

1. I. O. ANGell, "A table of totally real cubic fields," Math. Comp., v. 30, 1976, pp. 184-187.

2. H. DavenPort \& H. HeilbronN, "On the density of discriminants of cubic fields," Bull. London Math. Soc., v. 1, 1969, pp. 345-348.

3. H. J. Godwin \& P. A. Samet, “A table of real cubic fields," J. London Math. Soc., v. 34, 1959, pp. $108-110$.

4. P. Llorente, Cuerpos Cúbicos y Cuerpo de Clases de Cuerpos Cuadráticos Reales, Acta $2^{\circ}$ Congr. Mat., Venezuela, 1979.

5. P. Llorente \& A. V. Oneto, Cuerpos Cúbicos, Cursos, Seminarios y Tesis del PEAM, No. 5, Univ. Zulia, Maracaibo, Venezuela, 1980. 